\title{
A Novel System for Generating Multicultural Business Models
}

\author{
Manabu Suehiro*, Reiko Hishiyama \\ Faculty of Science and Engineering Waseda University, Tokyo, 169-8555 Japan. \\ * Corresponding author. Tel.: +81-80-2052-1135; email: msuehiro@asagi.waseda.jp \\ Manuscript submitted October 12, 2016; accepted March 18, 2017. \\ doi: 10.17706/ijeeee.2017.7.1.24-35
}

\begin{abstract}
These days, Japan is facing the necessity of developing new business models for non-Japanese persons. However, it is difficult to develop products or services that consider other countries' cultures and values. Our research task was to support companies developing new products or services that consider the viewpoints of non-Japanese persons by working together with them. We developed and in this paper propose a system that enables people with different mother tongues to participate in developing business models on the assumption that the participants are in distant places. We asked groups of people to develop new business models using the system as an experiment. The results showed that business models developed by groups with Japanese and Korean students were evaluated as comparable to models developed by groups comprising only Japanese students. The results indicate the proposed system can be used to develop new business models that take non-Japanese persons' viewpoints into account.
\end{abstract}

Key words: Business model, intercultural collaboration, machine translation, language grid.

\section{Introduction}

The number of tourists and business people visiting Japan is increasing these days and bringing huge business chances to the country. In addition, the Olympics will be held in Tokyo in the summer of 2020. This will involve a large amount of money to provide facilities for sport events, infrastructure for transportation, accommodations for the contingent of athletes, and maintenance. The economic impact of the 2012 London Olympics was reported in [1] and the Tokyo Olympics can also be expected to have a large economic impact.

On the other hand, Japanese companies are under the pressure of needing to develop new business models for non-Japanese persons. However, it is difficult to create new products or services that consider other countries' cultures and values since there are not many chances for people in Japanese companies to communicate with persons from other countries and exchange ideas with them. In many cases they can use English as a communication tool, but communicating in other languages is difficult. To overcome these problems, it is essential to provide people with the opportunity to exchange ideas in their native language.

To address this point, we created a multi-language thinking support system that has a machine translation function for effectively creating business models for non-Japanese persons by using their native languages. Using this system, we carried out experiments to ask pairs of students to develop new business models. We used the results obtained to analyze and evaluate business models and communication activities.

\section{Related Work}


It is said to be difficult to create new business models that have high novelty and feasibility. Accordingly, many types of frameworks have been proposed to create such business models more effectively in the field of business administration. One of them is called "Business Model Canvas (BMC)"[2], [3] and was designed by Osterwalder and his associates. This framework is used in many countries such as the United States. It divides business into nine blocks and business models can be expressed by filling in all blocks using post-it notes. The nine blocks are "Key Partners", "Key Activities", "Key Resources", "Value Proposition", "Customer Relationships", "Customer Segments", "Channels", "Revenue Streams", and "Cost Structures."

However, BMC was developed as a paper-based framework using only one language such as English. Consequently, problems relating to language and distance occur in adopting the opinions of persons whose native tongue is not the language used in the moment.

On the other hand, necessity of using Information and Communication Technology (ICT) has increased due to the development of the Internet in recent years. Of course in the field of economics. For example, an architecture modeling language called "Archimate" was developed in order to support modeling business, application, and technology architecture using computer [4]. It is used to describe, analyze, and visualize the business model. However, this modeling language is also used in only one language.

To use these tools, users are to communicate using only one language. It is not a problem if all users have same mother tongue. However, communication using a foreign language is difficult for many people. The language barrier prevents them from understanding other persons completely without misunderstandings [5], [6]. Machine translation is one technique used to overcome this problem [7].

Related work has identified three key points.

1) Visualization of a framework is useful for building a business model.

2) Using ICT is useful for building a business model.

3) Multilingualization using machine translation is useful for intercultural collaboration.

On the basis of these two points, we constructed a system for building a business model as a framework for multiple languages. We adopted the Business Model Canvas as an existing framework.

\section{Proposal}

Our aim was to support companies developing new products or services that consider the viewpoints of non-Japanese persons by working together with them. As a means to achieve this aim, we propose a system that enables people with different mother tongues to participate in developing business models on the assumption that the participants are in distant places. We asked pairs of students to use the system in experiments to develop new business models and analyzed and evaluated the results.

This system is an online application built by using PHP, HTML, MySQL, and jQuery. It allows users to chat and add post-it notes in their native languages by connecting to "Language grid [8]." This system has high diversity and flexibility since users can decide the pattern of service cooperation and service construction by linking with machine translation service. It also provides high versatility by managing canvas and post-it independently as a layer. The system's interface and structure are shown in Fig. 1 and 2.

System users perform the following actions.

1) Select a language and log in at the login screen.

2) Discuss a business model using the multi-language chat function.

3) Add post-it notes to the canvas.

With the multi-language chat function, users can send and check messages in the language selected at the login screen. In addition to the translating function, the chat system has a back-translation function that allows users to check how their messages are transmitted to other users [9]. The function translates user A's message into user B's language and then translates user B's language back into user A's language. This 
helps users to estimate how their messages will be transmitted to other users and fixes the original message in order to transmit users' ideas to other users.

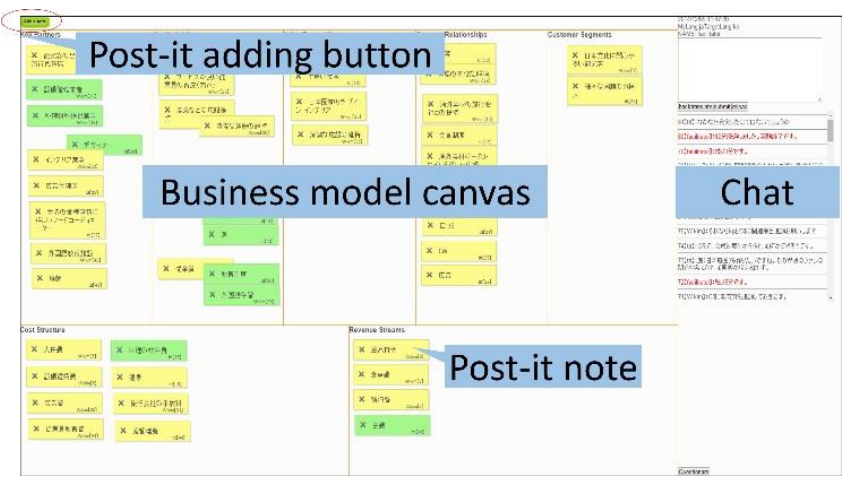

Fig. 1. System interface (language: Japanese).

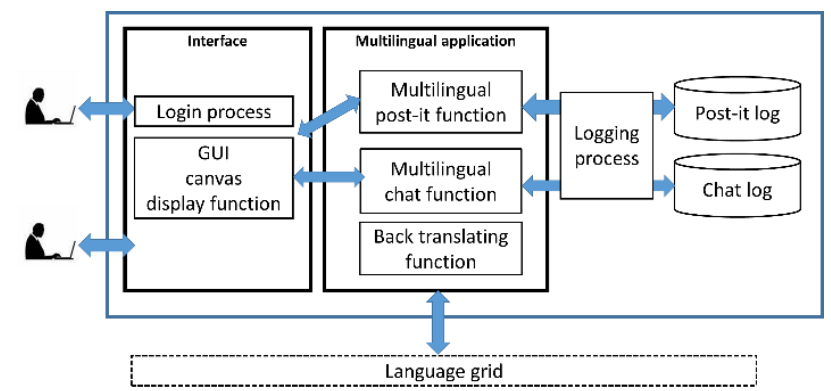

Fig. 2. System structure.

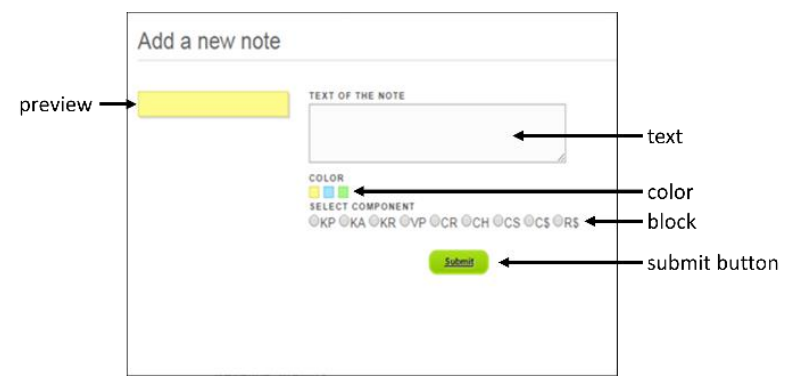

Fig. 3. Interface for adding post-it notes.

There is an "Add" button at the upper left of the interface. If the user clicks this button, a new interface (shown in Fig. 3) will be shown on the screen. Users can add post-it notes to the canvas by inputting information about the notes. All post-it notes made by users are translated into the users' selected language.

Users use these two functions to create business models, using an order of 2 or 3 at their discretion.

Comparison of paper-based BMC and proposed system is shown in the Table 1.

Table 1. Comparison between Paper-Based and Proposed System

\begin{tabular}{|c|c|c|}
\hline & Advantages & Disadvantages \\
\hline Pape-based BMC & $\begin{array}{l}\text { - Face to face } \\
\text { - No time lag }\end{array}$ & $\begin{array}{l}\text { - Only in single language } \\
\text { - Only in single place } \\
\text { - Difficult to storage }\end{array}$ \\
\hline Proposed system & $\begin{array}{l}\text { - Online } \\
\text { - Easy to storage (logging) } \\
\text { - Multilingual }\end{array}$ & $\begin{array}{l}\text { - Possibility of mistranslation } \\
\text { - Chat only }\end{array}$ \\
\hline
\end{tabular}


There are few disadvantages of using our system. However, advantages that our system provides exceed disadvantages and it will support developing business models in better way than paper-based BMC.

\section{Experiments}

We carried out experiments using the system. There were two types of experimental groups: "same language pair" groups comprising two Japanese students and "different language pair" groups comprising one Japanese student and one Korean student. The experiment was carried out eight times for each group. Incidentally, the theme of the business model in this experiment was "Accommodating foreigners visiting Japan."

The three steps in the experiment flow were as follows.

1) Thinking about the business model of a fast food shop for practice (15 minutes)

In this step, we explained the Business Model Canvas to the students and assigned them a practice problem, i.e., to think about a business model for a fast food shop in a limited amount of time. The goal was to enable them to understand how to use the Business Model Canvas.

2) Thinking about a business model for accommodating foreigners visiting Japan (60 minutes)

In this step, we explained the multi-language chat system and how to use it to the students. They were then asked to use the system to create business models by discussing the topic with each other in a limited amount of time.

3) Answering a questionnaire

At the end of the experiment, we asked the students to answer a questionnaire.

\section{Results and Discussion}

\subsection{Evaluation Results}

We evaluated the business models developed in the experiments on a scale of one to five using the evaluation criteria cited in [2]. The evaluation results are shown in Table2. Experiments numbered 1-8 in the table were done by the same language pair groups and those numbered 9-16 were done by the different language pair groups. The rows marked in gray are the models developed by the groups that we evaluated most highly. Table 3 shows the average evaluation results.

Table 2. Evaluation of Business Models (Unit: pt)

\begin{tabular}{l|c|c|c|c|c}
\hline \hline & Value proposition & Revenue and Cost & Infrastructure & Customer interface & Mean \\
\hline No.1 & 2.89 & 2.95 & 2.27 & 2.81 & 2.73 \\
No.2 & 4.44 & 3.67 & 3.83 & 3.54 & 3.87 \\
No.3 & 3.78 & 3.33 & 3.73 & 3.57 & 3.60 \\
No.4 & 4.06 & 3.29 & 3.10 & 2.54 & 3.24 \\
No.5 & 3.94 & 3.52 & 3.40 & 3.22 & 3.52 \\
No.6 & 3.89 & 3.29 & 3.67 & 2.81 & 3.41 \\
No.7 & 3.11 & 3.19 & 2.63 & 2.44 & 2.84 \\
No.8 & 3.28 & 2.57 & 2.38 & 1.98 & 2.55 \\
No.9 & 4.39 & 3.67 & 3.60 & 3.65 & 3.83 \\
No.10 & 4.00 & 3.86 & 3.63 & 3.48 & 3.74 \\
No.11 & 4.17 & 3.38 & 3.83 & 3.04 & 3.60 \\
No.12 & 4.28 & 3.38 & 3.77 & 4.15 & 3.89 \\
No.13 & 4.00 & 3.07 & 3.27 & 3.37 & 3.43 \\
No.14 & 4.50 & 3.86 & 3.67 & 3.89 & 3.98 \\
No.15 & 3.33 & 3.48 & 2.70 & 3.04 & 3.14 \\
No.16 & 3.67 & 3.02 & 2.23 & 3.19 & 3.03 \\
\hline \hline
\end{tabular}

1) Value proposition evaluation (3 categories) 
2) Revenue and cost evaluation (7 categories)

3) Infrastructure evaluation (5 categories)

4) Customer interface evaluation (9 categories)

Table 3. Evaluations for Experimental Groups

\begin{tabular}{c|l|c|c}
\hline \hline Category & Statistic & Same language & Different language \\
\hline \multirow{4}{*}{1} & Mean & 3.646 & 4.043 \\
\cline { 2 - 4 } & S.D. & 0.5781 & 0.3878 \\
\cline { 2 - 4 } & P-value & \multicolumn{2}{|c}{0.065} \\
\hline \multirow{4}{*}{2} & Mean & 3.226 & 3.465 \\
\cline { 2 - 4 } & S.D. & 0.3402 & 0.3213 \\
\cline { 2 - 4 } & P-value & \multicolumn{2}{|c}{0.086} \\
\hline \multirow{3}{*}{3} & Mean & 3.126 & 3.338 \\
\cline { 2 - 4 } & S.D. & 0.6288 & 0.5772 \\
\cline { 2 - 4 } & P-value & \multicolumn{2}{|c}{0.248} \\
\hline \multirow{3}{*}{4} & Mean & 2.864 & 3.476 \\
\cline { 2 - 4 } & S.D. & 0.5547 & 0.4016 \\
\cline { 2 - 4 } & P-value & \multicolumn{2}{|c}{$0.012^{*}$} \\
\hline \hline
\end{tabular}

The results showed the business models that the different language pair group had developed were evaluated slightly higher than those the same language pair group had developed. However, the results indicate that our system enables participants with different native languages to create new business models that take foreigners' points of view into account. The evaluation results are detailed below.

1) Value Proposition

The difference in the average evaluation values between the two types of groups was 0.397 and showed a significant $(p=0.065<0.10)$ tendency. When we increase the amount of data in the future we will in all probability obtain a more significant difference.

2) Revenue and Cost

The difference in the average evaluation values between the two types of groups was 0.239 and showed a significant $(p=0.086<0.10)$ tendency. Although the current framework does not consider the cost and revenue using specific values, the results show it is possible for different language pair groups to create reasonably good business models.

3) Infrastructures

The difference in the average evaluation values between the two types of groups was 0.212 , which shows that there is no significant difference between the two types of groups.

4) Customer Interface

The difference in the average evaluation values between the two types of groups was 0.239 , which shows that there is a significant difference between the two types of groups under the critical p-value, which is $5 \%$. The results show that the "different language pair" groups were able to consider the customer interface better than the "same language pair" groups. In the "different language pair" groups, adopting the Korean student's ideas made it possible to adopt a foreigner's point of view into the new business model.

\subsection{Creating Post-It Notes}

Fig.4 shows a scatter diagram of the evaluation results and the number of post-it notes. The correlation coefficient was 0.60 . The results show that there is moderate positive correlation between the two values and that adding meaningful post-it notes makes better business models. Meaningful post-it notes are those posted on the interface at the end of the experiment and do not include post-it notes deleted during the experiments. 


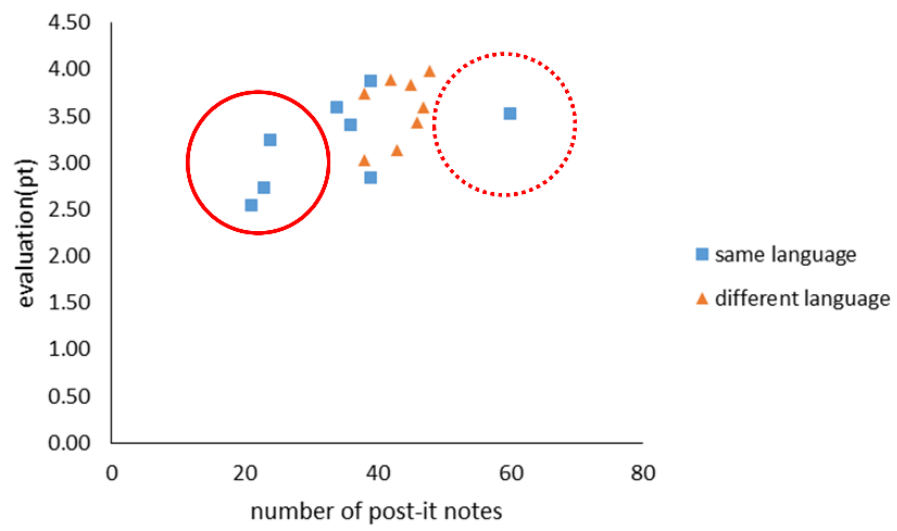

Fig. 4. Correlation between post-it notes and evaluation $(n=16)$.

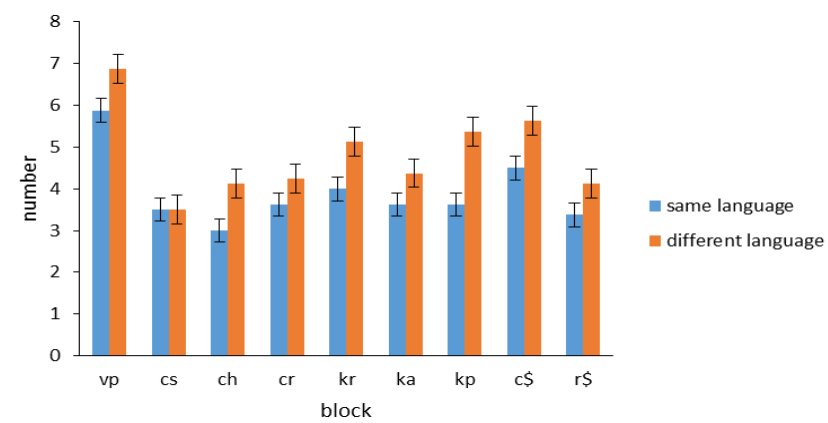

Fig. 5. Number of post-it notes sorted by block $(n=16)$.

Table 4. Number of Post-It Notes Added

\begin{tabular}{l|c|c}
\hline \hline & Same language & Different language \\
\hline Mean & 34.5 & 43.4 \\
\hline S.D. & 12.64 & 3.85 \\
\hline P-value & \multicolumn{2}{|c}{$0.047^{*}$} \\
\hline \hline
\end{tabular}

It is also possible to say that the scatter the "different language pair" groups produced was relatively low compared to that produced by the "same language pair" groups. It is also clear that the preceding discussion is relevant to the number of post-it notes. An example is the solid line circle and the dotted line circle in Fig. 4. The three pairs in the solid line circle show cases where post-it notes were added after discussing; the pair in the dotted line circle shows the case where post-it notes were added before discussing. It is conceivable that the valance of adding post-it notes and discussion is important.

Table 4 shows the difference in the number of post-it notes added by the "same language pair" and "different language pair" groups.

The difference in the average number of post-it notes between the two types of groups was 8.9, which shows that there is a significant difference between the two types of groups under the critical p-value, which is $5 \%$. The "different language pair" groups were able to add a certain number of meaningful post-it notes by adopting the Korean students' ideas. On the other hand, the "same language pair" groups, which comprised Japanese students who had studied abroad, were able to consider the viewpoints of non-Japanese persons. They were not, however, able to adopt specific ideas presented by such persons.

Fig. 5 is a comparison of the average number of post-it notes for each block. There was no major difference between the two types of groups, but the "different language pair" groups posted more post-it notes than the "same language pair" groups in the "Key Partners" block. It should be noted here that the "Key Partners" block was for thinking about partners and suppliers who constructed business models. Both 
types of groups came up with ideas such as "travel agent" and "advertising company". The "different language pair" groups also came up with ideas such as "internet user" and "famous blogger." These ideas came from the experiences of the Korean students, who before going abroad to study had studied about Japan from information they had obtained from travel agents and other Internet users.

For the "Channels" block, which shows how values should be noticed and provided to customers, three "same language pair" groups and six "different language pair" groups came up with the ideas "blog" and "word of mouth". This is due to the experience obtained from gathering information about foreign countries through the Internet. Three "same language pair" groups were able to come up with the same ideas as six "different language pair" groups because one or both of the students in the former had studied abroad while the students in the other "same language pair" groups were not able to do so. From this we deduce that using our system enables users to adopt their experiences and values into ideas. It is also possible that using our system will enable users to adopt foreigners' ideas to develop new products or services that take different cultures and values into account.

\subsection{Relationship between Chats and Post-It Notes}

In our system, users are only able to use multi-language chat function and add a post-it function to communicate with each other. Accordingly, we analyzed the relationship between chats and post-it notes by using logs for both. First of all, there two ways to use the post-it function as a communication tool.

- Showing decision after discussion

- Item Showing one's idea

In addition, it can be supposed that a discussion starts when one of the users proposes his or her idea. In that case, there are two ways to come up with a decision: the proposed idea becomes the decision or it is revised into the decision. Table 5 is the results of tagging using classification from the ways of using the post-it function as a communication tool. Fig. 6 shows the classification results. Here, "ratio" is the ratio of post-it notes that were discussed.

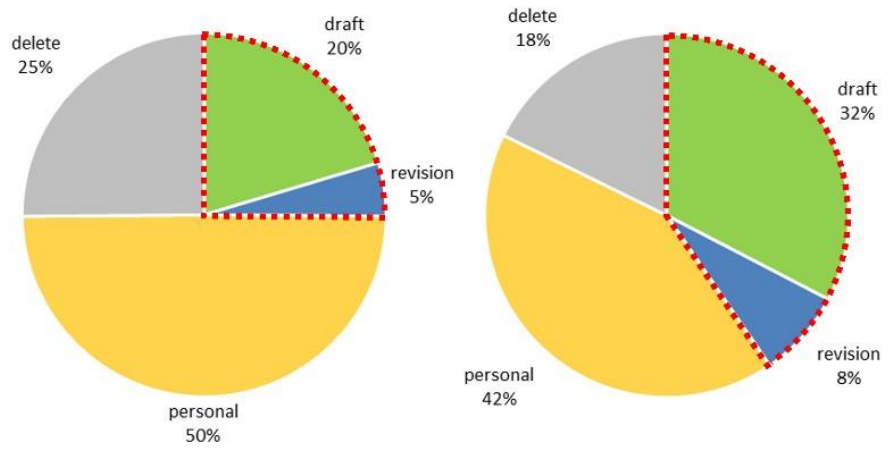

Fig. 6. Ratio of post-it notes.

Table 5. Types of Post-It Notes

\begin{tabular}{l|l}
\hline \hline Type & Definition \\
\hline Draft & Proposed idea becomes the decision \\
Revision & Proposed idea is revised into the decision \\
Personal & Post-it notes that were not discussed at all \\
Delete & Post-it notes that were deleted \\
\hline \hline
\end{tabular}

Regardless of the experimental group type, the proposed idea tended to become the decision when a discussion took place. However, it is clear that the discussion ratio is below $50 \%$ as shown in Fig. 6 . Some of the groups, such as for experiment no. 5, did not discuss anything at all and added post-it notes as the group 
opinion. As shown in Table 5, "Draft" and "Revision" have a relationship with chats. If the discussion ratio is low, it means that the pair only had a few discussions about the post-it notes they created. In the Business Model Canvas, deliverables are post-it notes created on the canvas and it is desirable that they are created after active discussion by users. In order to do so, it is necessary to set up some constraints. For example, one constraint is that users must discuss post-it notes created by one of the users at least once using a chat. However, this constraint makes it impossible to finish the Business Model Canvas because there is a time limit in this experiment. We must find a way to solve this dilemma in the future.

\subsection{Business Model Case}

In this section, we will examine business models that gained high evaluation. Fig. 7 and 8 show the business models that gained the highest evaluation in each experimental group. In both figures, post-it notes are surrounded by a solid line and a dotted line shows that they are related to each other.

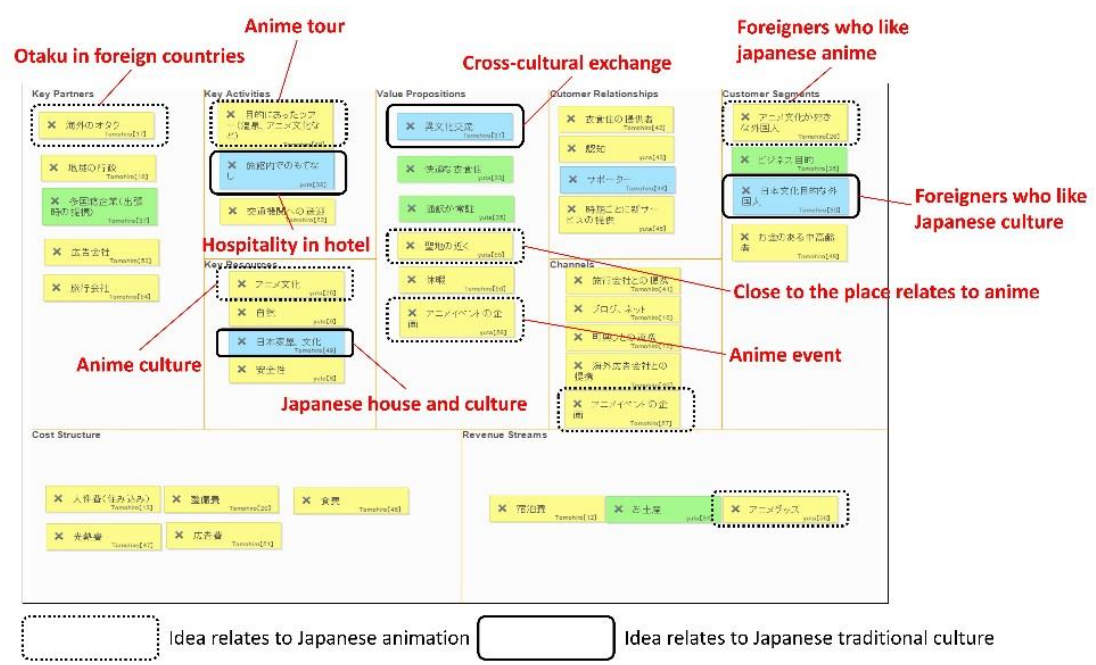

Fig. 7. Business model created in experiment no.2.

In experiment no.2, participants started creating business models by determining three "Customer Segment" and "Value Proposition" values corresponding to "Customer Segment" values they had determined. First, they determined the three "Customer Segment" values in accordance with the reason visitors came to Japan: to study Japanese animation, to study Japanese culture, or to conduct business. Next they determined at least one "Value Proposition" value that corresponded to each "Customer Segment" value. They determined representative "Value Proposition" values so as to correspond with Japanese animation events, cross-cultural communication, and interpreting environment preparations. They then completed their business models by finalizing details during discussions that focused on their "Customer Segment" and "Value Proposition" values. Our evaluation of their business models is shown below. Here, category $1=4.44 \mathrm{pt}$, category $2=3.67 \mathrm{pt}$, category $3=3.83 \mathrm{pt}$, category $4=3.54 \mathrm{pt}$, and the average score was $3.87 \mathrm{pt}$.

The participants in experiment no.14 also started creating business models by determining "Customer Segment" values. The chat log results indicate they agreed to narrow down "Customer Segment" values to those for wealthy Chinese and Korean persons in the 20-40 age range. After they had determined the "Customer Segment" values, they discussed "Value Proposition" values in the same way as the previously noted pairs. The main idea of "Value Proposition" values concerns the taste of Japanese culture they had acquired. The concept of their business models was remarkable. They included the idea of arranging two types of rooms, one expensive and one reasonable, in order to satisfy both "Customer Segment" values. Our 
evaluation of these business models is shown below. Here, category $1=4.50 \mathrm{pt}$, category $2=3.86 \mathrm{pt}$, category $3=3.67 \mathrm{pt}$, category $4=3.89 \mathrm{pt}$, and the average score was $3.98 \mathrm{pt}$.

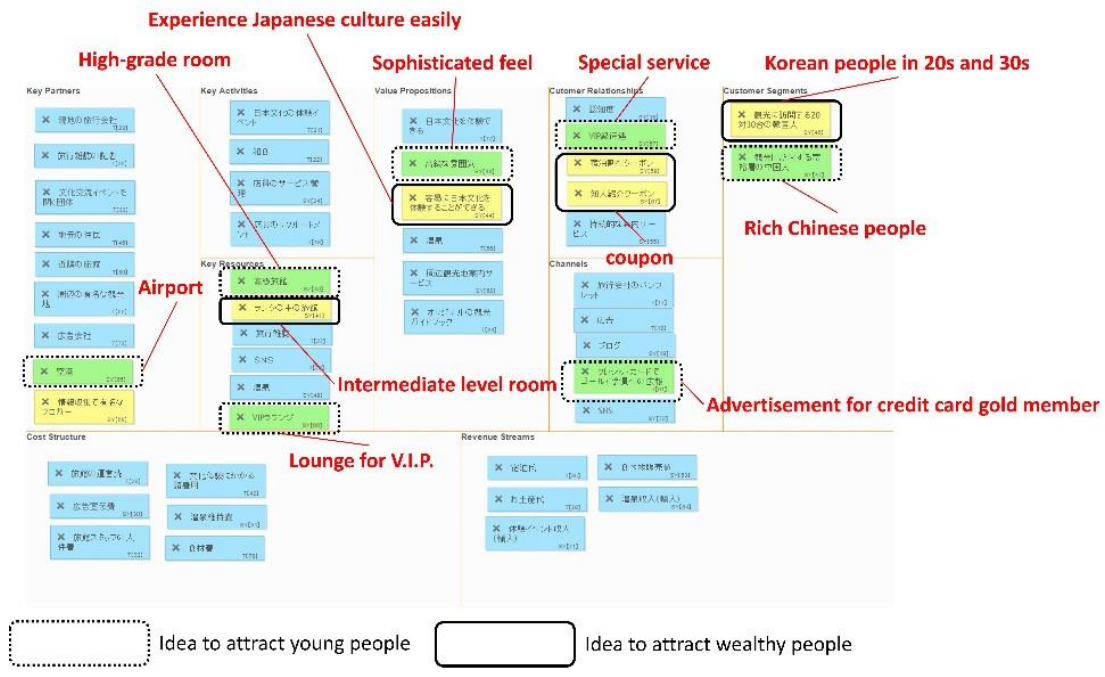

Fig. 8. Business model created in experiment no.14.

These two experiment results lead us to conclude that "Customer Segment" and "Value Proposition" values must be considered at the outset in order to create good business models. We also conclude that it is important to consider at least one original business model concept to differentiate models from those for other companies. We believe that adopting foreigners' ideas is a meaningful way to think about original business model concepts.

\subsection{Experiment Procedures}

In this section, we will describe how post-it notes were added and discussed during the limited experiment time. As we said in Section 3, participants can use their discretion in using the system to create business models. Accordingly, participants used three ways to complete the business models:

1) First discuss and then add post-it notes

2) First add post-it notes and then discuss

3) Add post-it notes while discussing

Table 6 shows the procedures the pairs used to create a business model using the system.

Table 6. Experiment Procedures

\begin{tabular}{c|c|c}
\hline \hline Process & Same language & Different language \\
\hline 1 & 2 pairs & 1 pair \\
2 & 1 pair & 1 pair \\
3 & 5 pairs & 6 pairs \\
\hline \hline
\end{tabular}

The table results show that many pairs chose method 3, i.e., adding post-it notes while discussing. We believe that the limited time made participants choose this method in order to keep a balance between adding post-it notes and discussion.

Graphs in Fig.9 show the average number of post-it notes and per minute utterances for each type of group. The averages were calculated using two pairs whose evaluation results were highest in their group. In the graphs, the horizontal axis is elapsed time and the vertical axis is the number of post-it notes and utterances. The results shown in the two graphs make it clear that pairs who received high evaluation results added post-it notes while discussing regardless of the type of group they were in. Specifically, we 
found that both experimental groups only discussed without adding post-it notes in the first five minutes. In this case, two types of discussions took place: discussions about the strategy of creating a business model and the concept of their business model for the given task. The strategy for creating a business model was how to complete the Business Model Canvas. After the first discussion the participants added post-it notes while discussing details for about thirty minutes. In the latter half of the experiment, details of the business model were determined and more post-it notes were added than in the first half. We believe that in order to create a business model that has a high degree of completion in the limited time, it is necessary to determine the complete picture of the business model in the first half and add details in the latter half.
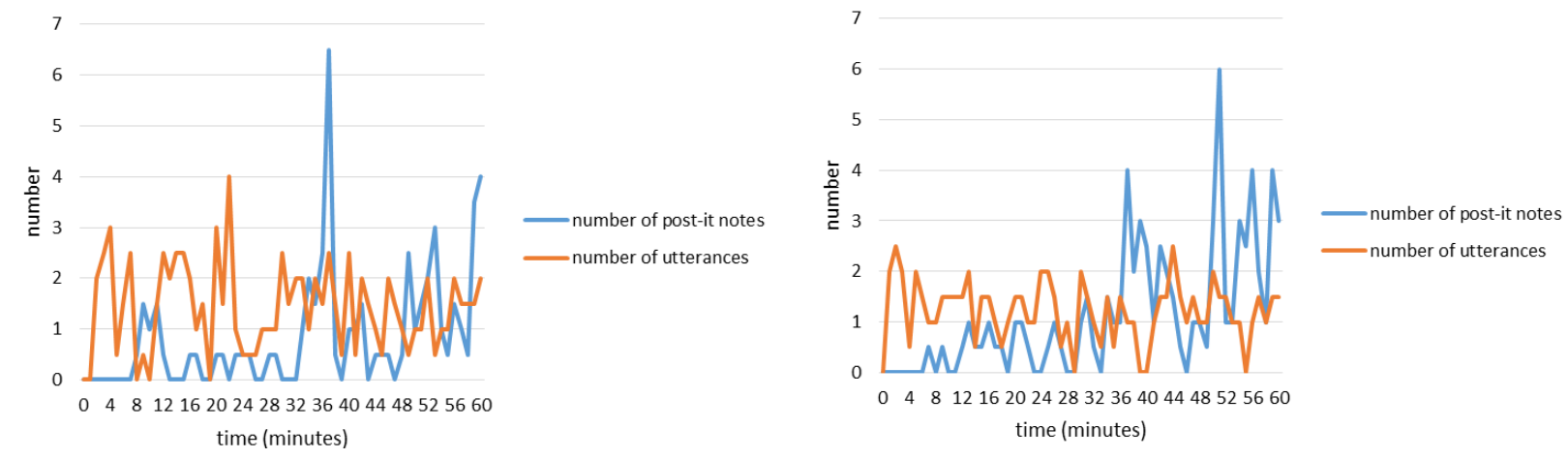

Fig. 9. Number of post-it notes and utterances (left: same language, right: different language).

\subsection{Investigation of Mistranslation}

There is a possibility of mistranslation due to the limited accuracy of current machine translation and that it might disturb communication between two participants in the experiment. Therefore, we surveyed the existence of mistranslation errors using a questionnaire. Fig. 10 shows the questionnaire results obtained. The only target in this case was "different language pair" groups since the "same language pair" groups did not use machine translation in the experiment.

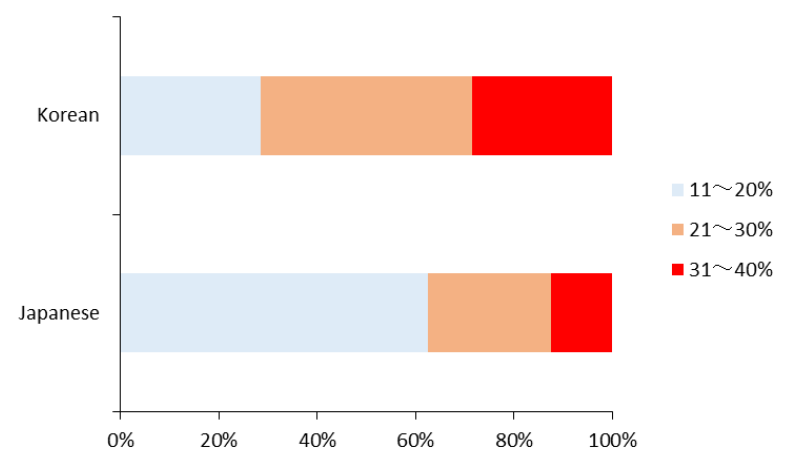

Fig. 10. Extent of mistranslation $(\mathrm{n}=16)$.

There were obviously some mistranslations in the experiment since no participants answered that the percentage of mistranslation was only 0 to 10 percent. On the other hand, no participants answered that the percentage of mistranslation was 41 to 100 percent, which shows that mistranslation played a minimal part in inter-participant communication. In addition, actions to overcome mistranslation such as that shown in Table 7 were observed. In the experiments, Japanese participants could not understand the meaning of post-it notes added to "Revenue Streams" by Korean participants because of mistranslation. However, the 
Korean participants explained the meaning of the post-it notes by using examples, which enabled the Japanese participants to finally understand the meaning. Thus, the Korean and Japanese participants solved the mistranslation problems by conversing with each other. This indicates the system is practical when the languages spoken are Japanese and Korean. For other languages, however, the system's practicality is not clear and this is a topic for further study.

Table 7. Actions to Overcome Mistranslation

\begin{tabular}{l|l|l}
\hline \hline & original text in Japanese & text in English \\
\hline Korean & R\$に記念品も追加してみます。 & I will add "present" to R\$. \\
Japanese & すみません、記念品とは何ですか ? & Excuse me. What's "present"? \\
Korean & 東京バナナ等等 & Such as "Tokyo banana" \\
Korean & 翻訳にこ問題があるようです。 & There is a problem in translation. \\
Japanese & おみややげみたいなことですね ! & Like souvenir! \\
Japanese & 理解しました ! & I understood! \\
\hline \hline
\end{tabular}

In the research of Terui [10], mistranslation problems were solved by the intervention of a facilitator who, whenever such a problem occurred, asked the speaker what he or she wanted to say. In our research, the facilitator intervened only to activate discussion between participants. To facilitate effective discussions among participants in the limited amount of time, the facilitator plays an important role in clarifying mistranslated sentences as well as in activating discussions.

\subsection{Practical Use}

In this section, we will think about how to make full use of our system in creating new business models. Since experiments showed the efficacy of adopting the ideas of non-Japanese persons, asking those persons to join as participants is the most important thing in using our system. We should also employ persons from many other countries besides Japan since non-Japanese persons provided considerable information in the experiments.

Let us start with the idea that having no specific concept of a new business model might create a situation in which the limited discussion time is used up before truly meaningful discussions can be held. As we said in 5.4, discussing important things about business models such as the concept and specific ideas is very important to start creating new business models. We also believe that comparing business models made by many kinds of groups will be fruitful in finding new business model concepts.

\section{Conclusion}

In this paper, we explained how we created a multi-language thinking support system in order to effectively create business models for non-Japanese persons. We took a new approach by converting a previous method of creating business models into a system that could be carried out online for multiple languages.

Experiment results obtained for two types of experimental groups using the system showed that business models created by "different language pair" groups were evaluated almost as highly as models created by "same language pair" groups. This showed the possibility of using the system to adopt foreigners' ideas into new business models. The results also showed that the system is practical for cases in which the spoken languages are Japanese and Korean. We also extracted rules for creating business models using this system by observing business models created in experiments.

In future work, it will be necessary to think about how to use our system under different conditions such as the languages used and the number of participants. It will also be necessary to adopt certain rules to create good business models. This is because no rules were used in this research and as a result the 
evaluation of the business models was inconsistent. Those rules will need to be adopted by using the data obtained in this research. It will also be important to use foreigners other than Koreans as experiment participants to bring us new knowledge.

\section{Acknowledgment}

This work was supported by a Grant-in-Aid for Scientific Research (S) $(24220002,2012-2016)$ from the Japan Society for the Promotion of Science (JSPS).

\section{References}

[1] Blake, A. (2005). The Economic Impact of the London 2012 Olympics. Discussion paper: Christel DeHaan Tourism and Travel Research Institute: Nottingham University Business School.

[2] Osterwalder, A., \& Pigneur, Y. (2010). Business Model Generation. Wiley.

[3] Strategyzer. (2015). Create value for your business. Retrieved from the website: https://strategyzer.com/canvas

[4] Iacob, M., Jonkers, H., Lankhorst, M., \& Proper H. (2009). ArchiMate 1.0 specification. Zaltbommel. Netherlands: VanHaren Publishing.

[5] Aiken, M. (2002). Multilingual communication in electronic meetings. ACM SIGGROUP, Bulletin, 23(1), 18-19.

[6] Quaddus, M. A., \& Tung, L. L. (2002). Explaining cultural differences in decision conferencing. Communications of the ACM, 45(8), 93-98.

[7] Yamashita, N., \& Ishida, T. (2006). Effects of machine translation on collaborative work. Proceedings of the 200620 th Anniversary Conference on Computer Supported Cooperative work, 515-524.

[8] Ishida, T. (2006). Language grid. An infrastructure for intercultural collaboration. IEEE/IPSJ Symposium on Applications and the Internet (SAINT-06), 96-100.

[9] Miyabe, M., Yoshino, T., \& Shigenobu, T. (2009). Effects of undertaking translation repair using back translation. Proceedings of the 2009 International Workshop on Intercultural Collaboration, 33-40.

[10] Terui, K., \& Hishiyama, R. (2013). Multilingual case method system for cross-cultural analysis. International Conference on Culture and Computing, 117-122.

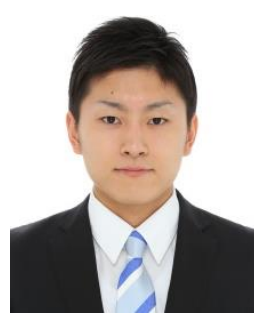

Manabu Suehiro is a student Graduate School of Creative Science and Engineering in Waseda University. He received his bachelor of engineering in 2015 from School of Creative Science and Engineering in Waseda University. His field of study is knowledge information processing especially analyzing user's action using machine translation.

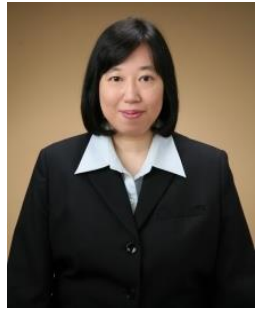

Reiko Hishiyama is a professor in the Graduate School of Creative Science and Engineering in Waseda University, where she directs the intelligent information system laboratory. She received her doctor of informatics in 2005 from Kyoto University. She taught at Kyoto Women's University, before joining the faculty of Waseda University. Her current research interests include artificial intelligence, autonomous multiagent systems, knowledge representation, autonomy-oriented computing and related areas. The topic is viewed as an interdisciplinary field where computer science intersects with organization science, sociology, psychology, and other fields. 